\title{
Descripción del uso y manejo de plaguicidas en las empresas de flores afiliadas a Asocolflores
}

\author{
Marcela E. Varona ${ }^{1}$, Jorge E. Tolosa ${ }^{2}$, Omayda Cárdenas ${ }^{1}$, Carlos H. Torres ${ }^{3,4}$, \\ Darío Pardo ${ }^{1}$, Gabriel Carrasquilla ${ }^{3,4}$, Howard Frumkin ${ }^{5}$ \\ ${ }^{1}$ Laboratorio de Salud Ambiental, Instituto Nacional de Salud, Bogotá, D. C., Colombia. \\ 2 Universidad Thomas Jefferson, Filadelfia, PA, Estados Unidos. \\ ${ }^{3}$ Asociación Colombiana de Exportadores de Flores, Bogotá, D. C., Colombia. \\ ${ }^{4}$ Facultad de Salud, Universidad del Valle, Cali, Colombia. \\ ${ }^{5}$ Emory University, Atlanta, GA, Estados Unidos
}

Introducción. En Colombia, la exposición a plaguicidas se ha convertido en un problema de salud pública debido al incremento de la demanda; esto ha ocasionado impacto en la salud de la población y en el ambiente.

Objetivo. Describir los plaguicidas empleados y determinar su uso y manejo en las empresas de flores en la sabana de Bogotá y Rionegro (Antioquia) afiliadas a la Asociación Colombiana de Exportadores de Flores, Asocolflores.

Materiales y métodos. Se realizó un estudio descriptivo en 84 empresas estratificadas por ubicación geográfica y por tamaño. Se recolectó información de la empresa y del trabajador, y se realizó una observación detallada del proceso de fumigación y utilización de los plaguicidas. Se llevó a cabo un análisis univariado, bivariado y la exploración de posibles asociaciones. Resultados. El 39,4\% de los trabajadores de las empresas incluidas en el estudio pertenecían al sexo masculino y el $60,6 \%$ al femenino. Respecto a la categoría toxicológica, el $14,3 \%$ de los plaguicidas pertenecía a la categoría I, el $14,4 \%$ a la II, el 52,0\% a la categoría III y el restante, $19,2 \%$, a la categoría IV. Los plaguicidas más utilizados fueron los ditiocarbamatos con 11,7\%. Los equipos más empleados para la aplicación de los plaguicidas eran la bomba móvil $(92,8 \%)$ y la lanza (92,9\%). El seguimiento de la exposición a plaguicidas por pruebas de laboratorio, se realizaba por medio de la colinesterasa mediante la técnica de Michel-Aldrige en el 85,9\% de las empresas.

Conclusión. Este estudio describe la forma como se utilizan los plaguicidas en la industria floricultora en Colombia. Se hacen recomendaciones para mejorar su manejo y se proponen medidas para reducir la exposición de los trabajadores.

Palabras clave: plaguicidas, uso de plaguicidas, toxicidad.

Pesticides use by flower companies associated with the Colombian Association of Flower Exporters

Introduction. In Colombia, pesticide exposure has became a public health problem, as the use of these substances continues to increase.

Objective. The current study accumulated information concerning pesticides used by flower companies in Bogotá and Rionegro (Antioquia) that were associated with Asociación Colombiana de Exportadores de Flores (Asocolflores) in Colombia.

Matherials and methods. Eighty-four companies were stratified by geographic location and size. Company and worker information was collected, and for each company, the process of pesticide application and maintenance of the cultivated flowers was carefully observed. Univariate and bivariate, and correlation analyses were applied for data analysis.

Results. Sex of workers was $39.4 \%$ male and $60.6 \%$ female. Pesticides were grouped into 4 toxicity classes: $14.3 \%$ were class I, $14.4 \%$ class II, $52.0 \%$ class III, and $19.2 \%$ class IV. Dithiocarbamates was the group of pesticides more commonly used (11.7\%). The equipment most frequently used for pesticide application was the "bomba móvil" (92.8\%), and the "lanza" 
(92.9\%). Cholinesterase activity measured by the Michel-Aldrige method was the biological marker for exposure to pesticides used in $85.9 \%$ of the companies.

Recommendations for improvements in their use and for measures to further reduce exposure of workers are made.

Keywords: pesticides, pesticides utilization, toxicity.

Las evidencias experimentales y epidemiológicas indican que ciertos químicos industriales y ambientales ubicuos, tanto en el ambiente ocupacional como en el general, pueden causar efectos adversos en los humanos. Entre estos químicos, los plaguicidas poseen especial importancia debido a su frecuente uso, el gran número, su amplia distribución medioambiental con vasta exposición humana y sus características toxicológicas (1-6).

En Colombia no existe suficiente información científica que nos permita determinar si la exposición a plaguicidas en las empresas de flores resulta en un efecto adverso sobre la salud de los trabajadores y sus familias. Entre los estudios realizados, Restrepo et al. -a comienzos de la década de los años 80- determinaron la prevalencia de efectos adversos sobre la reproducción en trabajadores de las empresas de flores y mostraron un moderado incremento de abortos, recién nacidos prematuros y malformaciones congénitas (7); otro estudio de casos y controles basado en el citado anteriormente, evaluó los efectos al nacer en los hijos de las madres ocupacionalmente expuestas a plaguicidas; se encontró un incremento de hemangiomas en niños cuyos padres estaban expuestos a plaguicidas en los cultivos de flores (8).

En 1996, el Instituto Nacional de Salud (INS) realizó un estudio de seguimiento en la cohorte de mujeres estudiada por Restrepo et al.; se encontró en la muestra del estudio una tasa de incidencia de cáncer menor a la reportada por el Registro Poblacional de Cáncer de Cali, el cual se considera como la fuente de información de incidencia de cáncer más confiable de Colombia (9).

\section{Correspondencia:}

Marcela Varona, Instituto Nacional de Salud, Tel. 220

7700 ext. 448, Fax 2207700 ext. 447, mvarona@ins.gov.co, Calle 26 No. 51-60.

Recibido: 03/02/05; aceptado: 01/07/05
Otro estudio realizado por el INS en 1999 evaluó la prevalencia de alteraciones citogenéticas en trabajadoras expuestas a plaguicidas en cultivos de flores de Bogotá; se encontraron frecuencias de alteraciones citogenéticas dentro de los valores de referencia reportados en la literatura (10).

La floricultura se inició en Colombia en los años 60 y se planteó como una actividad de exportación; el crecimiento ha sido rápido, y ocupa el segundo lugar en el mundo dentro de los países con mayores exportaciones de flores cortadas y se ha constituido en el renglón más importante de las exportaciones no tradicionales del país. Las altas exigencias sanitarias y estéticas del mercado internacional han hecho necesaria la aplicación de los conceptos del manejo integrado de plagas y enfermedades como parte del sistema de producción vegetal.

Tradicionalmente, en Colombia las empresas del sector floricultor se han ubicado en dos áreas geográficas específicas: la sabana de Bogotá y Rionegro (Antioquia). El sector cuenta con una asociación comercial, Asocolflores (Asociación Colombiana de Exportadores de Flores), la cual agrupa empresas tanto de la sabana de Bogotá como de Rionegro (Antioquia) y lleva a cabo un programa denominado Florverde, el cual trabaja por el mejoramiento continuo hacia el desarrollo sostenible, para perfeccionar las condiciones sociales y ambientales del sector; abarca aspectos de manejo de personal, salud ocupacional, bienestar y capacitación con un enfoque de sistema de gestión que apoya la planeación, ejecución, seguimiento y ajuste de los procesos de gestión humana.

Esta investigación se llevó a cabo teniendo en cuenta la posible asociación entre la exposición a plaguicidas y sus efectos sobre la salud (1113), la población expuesta, la dificultad en definir exposición ocupacional a plaguicidas y la importancia de realizar estudios para mejorar la clasificación de la exposición. 
Los objetivos de este estudio fueron describir los plaguicidas (herbicidas, insecticidas y fungicidas) empleados en las empresas de flores de la sabana de Bogotá y Rionegro (Antioquia) afiliadas a Asocolflores, determinar cómo se utilizan y aplican estos plaguicidas en los cultivos, describir el uso de elementos de protección personal y los patrones de tiempo de reentrada y de rotación de los trabajadores como actividades relacionadas con la exposición a plaguicidas.

\section{Materiales y métodos}

Este estudio se llevó a cabo en los años 2000 a 2002 en 84 empresas de flores ubicadas en la región de la sabana de Bogotá y de Rionegro (Antioquia). La selección de las empresas participantes en el estudio se hizo aleatoriamente a partir de un listado de 174 empresas registradas en Asocolflores. Las empresas de la muestra se estratificaron por ubicación geográfica en la sabana de Bogotá y Rionegro (Antioquia) y por tamaño, en pequeñas, menores de 50 trabajadores; medianas, iguales o mayores a 50 y menores o iguales a 200 trabajadores, y grandes, mayores de 200 trabajadores.

Para determinar el número de empresas necesarias para hacer una descripción representativa en el uso de plaguicidas del sector floricultor, se empleó la fórmula de muestreo aleatorio estratificado con asignación proporcional (14) según tamaño de la empresa, empleando una prevalencia de uso de plaguicidas categoría toxicológica I (extremadamente tóxicos) del 27\%, reportada por la Asociación Colombiana de Exportadores de Flores, Asocolflores (enero-octubre, 1999) y una amplitud del intervalo de prevalencia de $\pm 5 \%$, esperando encontrar una estimación entre el 22 y el $32 \%$ para la proporción de uso de plaguicidas.

El tamaño de muestra calculado fue de 84 empresas, de las cuales 75 estaban ubicadas en la sabana de Bogotá y 9 en Antioquia. La distribución fue proporcional según el tamaño de la empresa; en la sabana de Bogotá, 4 en la categoría de empresas pequeñas, 34 medianas y 30 grandes; en Antioquia, las empresas se distribuyeron en 3 medianas y 5 grandes.

Las empresas fueron contactadas a través de Asocolflores con el fin de dar a conocer los objetivos y los beneficios de la investigación; igualmente, se les explicó el mecanismo para asegurar la confidencialidad de la información de cada finca y de cada trabajador participante en el estudio.

Para la recolección de la información se diseñaron tres formularios; el primero obtuvo información de las empresas sobre el número de trabajadores, oficios desarrollados, tiempos de reentrada, elementos de protección personal suministrados a los trabajadores, forma de aplicación de los plaguicidas y las pruebas de laboratorio realizadas a los trabajadores; un segundo formulario recolectó información del trabajador sobre oficio, uso de elementos de protección personal, tiempos de reentrada y algunos datos sobre la prueba de la acetilcolinesterasa. Antes de recolectar esta información, cada trabajador aceptó verbalmente participar, teniendo la certeza de confidencialidad en la información proporcionada a los investigadores y su anonimato ante los empleadores; el tercer instrumento fue un listado de verificación a través del cual el equipo de trabajo de campo realizó una observación detallada del almacenamiento y del uso del proceso de aspersión de los plaguicidas con el fin de determinar los métodos empleados en la preparación de mezclas y la distribución de los plaguicidas dentro del cultivo, los equipos para la aplicación, las medidas de higiene tomadas y los elementos de protección personal empleados para la reducción de la exposición de los trabajadores.

Toda esta información recolectada se comparó con las disposiciones sanitarias sobre el uso y manejo de plaguicidas dadas por el Ministerio de Salud en el Decreto 1843 de 1991 (15). Los tiempos de reentrada (periodo de tiempo en el cual el nebulizado está seco, el particulado segmentado y los vapores dispersos y tiene por objeto proveer un margen de seguridad a los trabajadores después de aplicaciones de fumigantes, nieblas, aerosoles o humos) se compararon con los establecidos por Florverde $(16,17)$, los cuales son los únicos existentes en el país, y los tiempos de rotación (tiempo asignado para realizar labores de almacenista, bombero o asperjador) únicamente se describieron ya que no existen estándares de comparación. 
La totalidad de los instrumentos se codificaron con el fin de asegurar la confidencialidad de la información. Para la prueba de estos instrumentos, se realizó un estudio piloto en tres empresas ubicadas en la sabana de Bogotá y se hicieron los ajustes correspondientes.

Las entrevistas las hizo un equipo de investigadores calificados y entrenados en el diligenciamiento y la aplicación de los instrumentos. Se seleccionaron aleatoriamente cuatro trabajadores por empresa; dos de riesgo alto (pertenecen a este grupo los operarios que manejan directamente agroquímicos), uno de riesgo medio (los operarios que no manejan directamente los plaguicidas) y uno de riesgo bajo en el que se encuentra el personal administrativo.

El uso de plaguicidas se cuantificó estimando el ingrediente activo en $\mathrm{kg} / \mathrm{hectárea}$, teniendo en cuenta que el indicador internacional para el consumo de productos fitosanitarios es kilogramos de ingrediente activo por hectárea por año $(\mathrm{kg}$ de $\mathrm{ia} / \mathrm{Ha} / \mathrm{año}$ ). Con esta medida se obtiene un parámetro sobre la cantidad de sustancias tóxicas y potencialmente contaminantes que se aplican por unidad de área en un año (16).

Se realizó una base de datos en MS Access 97 para Windows (Redmond, Washington, 1995) para cada instrumento. Para el análisis bivariado se utilizó Stata 6.0 (Stata Corporation, Washington). Todas las variables se estratificaron por ubicación y tamaño de la empresa.

El estudio fue aprobado por el Comité Técnico de Investigación y el Comité de Ética del Instituto Nacional de Salud.

\section{Resultados}

Se obtuvo información de 84 empresas, 8 más del tamaño inicial de la muestra (76); éstas sirvieron como reemplazo en los casos en que las seleccionadas inicialmente no correspondieran al número de trabajadores, lo que hizo necesario ajustar el tamaño de los estratos; quedaron 75 $(89,3 \%)$ ubicadas en la sabana de Bogotá y 9 $(10,7 \%)$ en Antioquia.

Las empresas se estratificaron por tamaño de la siguiente manera: en la sabana de Bogotá se estudiaron $34(40,5 \%)$ empresas grandes, 36 $(42,9 \%)$ medianas y 5 pequeñas $(5,9 \%)$, y en Antioquia, $6(7,1 \%)$ grandes y $3(3,6 \%)$ medianas.

El promedio de antigüedad de las empresas fue de 14,2 años (máximo=31, mínimo=4, DE=7). En la sabana de Bogotá, las empresas grandes reportaron un promedio de antigüedad en años de 14,7 , las medianas de 13,5 y las pequeñas de 16,2; en Antioquia, las empresas grandes mostraron un promedio de 14,9 y las medianas de 11,3 años de antigüedad.

Las empresas participantes informaron 23.029 trabajadores, de los cuales, $9.082(39,4 \%)$ pertenecían al sexo masculino y 13.947 (60,6\%) al femenino. El $67,4 \%$ de los trabajadores de Antioquia eran hombres mientras que, en la sabana de Bogotá, el 63,5\% de la fuerza laboral eran mujeres. Se encontró una diferencia estadísticamente significativa entre sexo y ubicación geográfica $(p<0,01)$. Del total de mujeres vinculadas a las empresas del estudio, 1.008 $(7,2 \%)$ estuvieron embarazadas en 1999 , información reportada por el área de recursos humanos de cada empresa. Los trabajadores de riesgo alto constituyeron el 5,0\% del total de la fuerza laboral de las 84 empresas participantes; los de riesgo medio, el $88,0 \%$, y los de riesgo bajo, el 7,0\%.

La figura 1 presenta el porcentaje de uso de los plaguicidas por categoría toxicológica y ubicación geográfica en 1999. En Colombia, las cuatro categorías establecidas son: categoría I: extremadamente tóxico; categoría II: altamente tóxico; categoría III: medianamente tóxico y categoría IV: ligeramente tóxico $(15,18)$.

Se encontraron diferencias estadísticamente significativas en la utilización de plaguicidas por categoría toxicológica al comparar las empresas grandes por zona del país (sabana de Bogotá o Antioquia), en la categoría toxicológica I y II ( $p=0,008$ y 0,04 , respectivamente); la categoría III demostró una tendencia hacia una diferencia estadísticamente significativa $(p=0,09)$ y no se encontró diferencia en la categoría IV $(p=0,145)$. Al comparar las empresas medianas por zona del país, se encontraron diferencias estadísticamente significativas en las categorías I $(p=0,001)$, II 


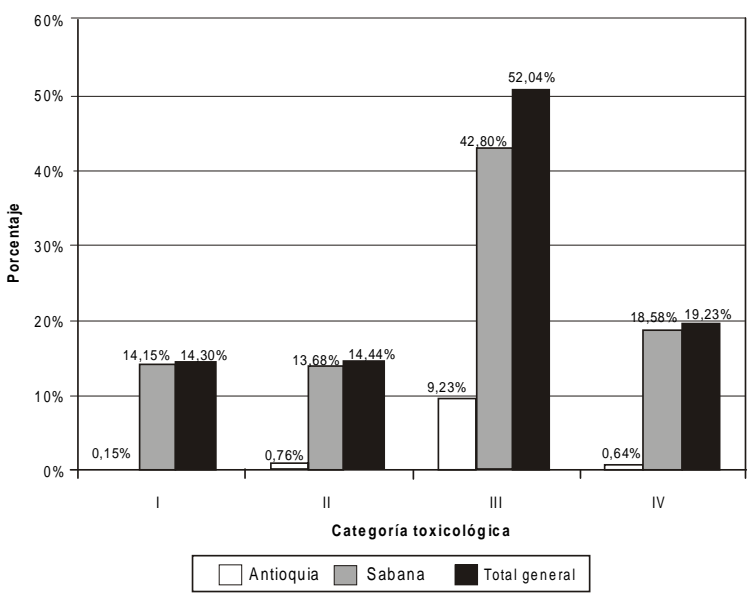

Figura 1. Porcentaje de uso de plaguicidas por categoría toxicológica y ubicación geográfica de la empresa, 1999.

( $\mathrm{p}=0,005)$ y III $(\mathrm{p}=0,004)$ pero no para la categoría IV $(p=0,331)$. Las empresas pequeñas no se compararon para esta variable ya que en Antioquia el muestreo no identificó empresas de este tamaño.

Al observar la distribución de plaguicidas por categoría toxicológica de forma independiente para cada área geográfica estudiada, se encontró que en la sabana de Bogotá el 47,98\% pertenecía a la categoría III, seguido por el $20,83 \%$ correspondiente a la categoría IV, el $15,86 \%$ a los plaguicidas de la categoría I y el $15,33 \%$ a los de categoría II. En Antioquia, el 85,59\% correspondió a los plaguicidas categoría III, seguidos por los de categoría II en $7,07 \%$, categoría IV por $5,96 \%$ y la categoría I con el 1,38\%.

La figura 2 muestra los plaguicidas de uso más frecuente por grupo químico en las 73 empresas de flores que reportaron el listado de plaguicidas para 1999, por ubicación geográfica.

La distribución de plaguicidas por grupo químico muestra que, en la sabana de Bogotá, los plaguicidas más utilizados son los ditiocarbamatos, seguidos por los hidrocarburos clorados más organoclorados y los metilisocianatos, mientras que en Antioquia, en primer lugar, están los metilisocianatos, seguidos por los benzimidazoles y los ditiocarbamatos.

Respecto a los periodos de rotación de los trabajadores de riesgo alto se presentan variaciones según el oficio desempeñado. El cuadro 1, muestra el promedio de días de rotación por oficio. Al comparar los periodos de rotación entre los asperjadores, los bomberos y los almacenistas tanto en Antioquia como en la

Cuadro 1. Periodos de rotación (días) de los trabajadores de riesgo alto según tamaño y ubicación geográfica de la empresa, Colombia, 2000.

\begin{tabular}{|c|c|c|c|c|c|}
\hline \multirow{2}{*}{$\begin{array}{l}\text { Tamaño } \\
\text { Ubicación } \\
\text { Oficio }\end{array}$} & \multicolumn{2}{|c|}{ Grande } & \multicolumn{2}{|c|}{ Mediana } & \multirow{2}{*}{$\begin{array}{c}\text { Pequeña } \\
\begin{array}{r}\text { Sabana } \\
(n=34)\end{array}\end{array}$} \\
\hline & $\begin{array}{c}\text { Antioquia } \\
(n=6)\end{array}$ & $\begin{array}{l}\text { Sabana } \\
(n=34)\end{array}$ & $\begin{array}{c}\text { Antioquia } \\
(n=6)\end{array}$ & $\begin{array}{c}\text { Sabana } \\
(n=34)\end{array}$ & \\
\hline \multicolumn{6}{|c|}{ Asperjador* } \\
\hline Promedio & 75,0 & 80,6 & 80,0 & 65,9 & 31,4 \\
\hline Máximo & 90 & 180 & 90 & 120 & 60 \\
\hline Mínimo & 60 & 15 & 60 & 7 & 7 \\
\hline $\mathrm{DE}$ & 16,4 & 32,6 & 17,3 & 32,6 & 18,8 \\
\hline \multicolumn{6}{|l|}{ Bombero } \\
\hline Promedio & 193 & 225,9 & 201,7 & 279,4 & NA \\
\hline Máximo & 365 & 365 & 365 & 365 & NA \\
\hline Mínimo & 60 & 30 & 60 & 30 & NA \\
\hline $\mathrm{DE}$ & 109,3 & 128,5 & 153,7 & 120,5 & NA \\
\hline \multicolumn{6}{|c|}{ Almacenista } \\
\hline Promedio & 319,2 & 342,3 & 365,0 & 365,0 & 365,0 \\
\hline Máximo & 365 & 365 & 365 & 365 & 365 \\
\hline Mínimo & 90 & 30 & 365 & 365 & 365 \\
\hline DE & 112,3 & 77,5 & 0,0 & 0,0 & 0,0 \\
\hline
\end{tabular}

* $p=(0,06)$; DE: desviación estándar 


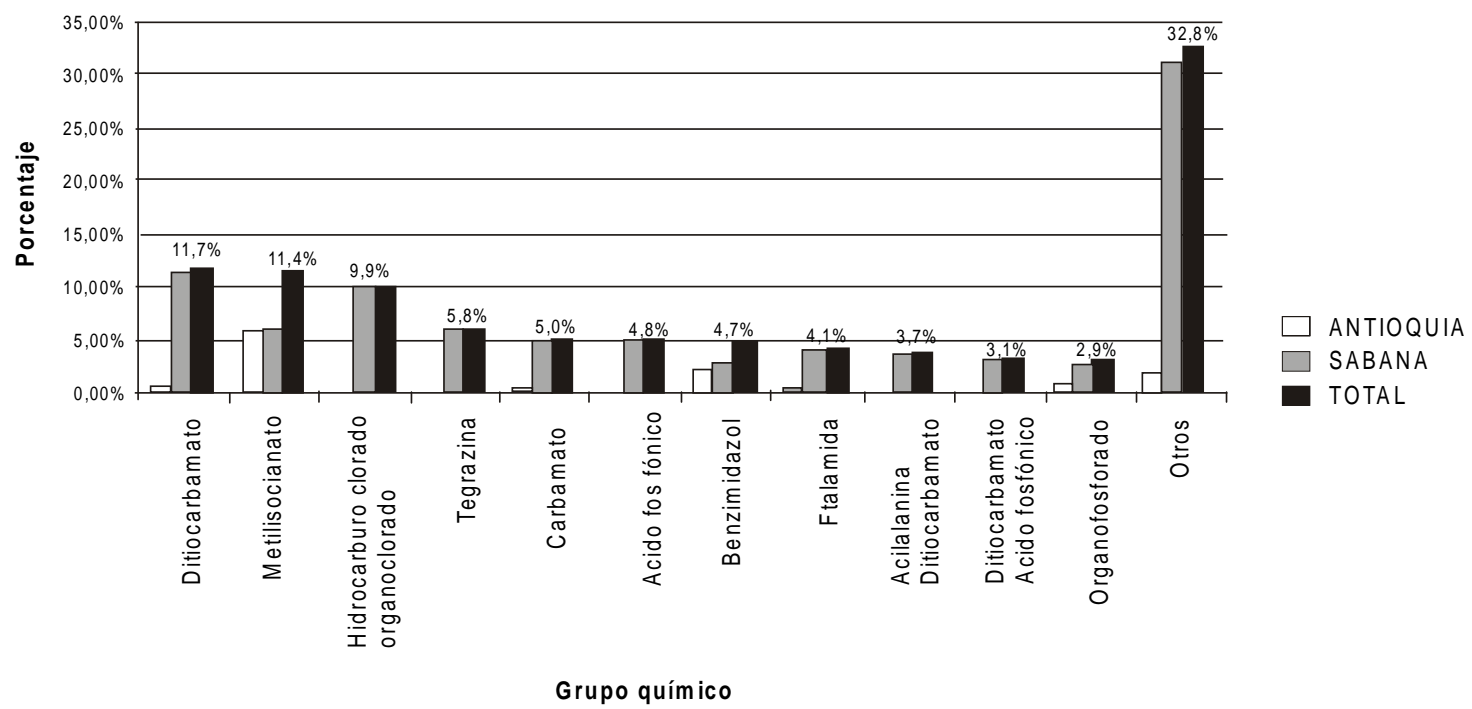

Figura 2. Porcentaje de uso de plaguicidas por grupo químico y ubicación geográfica de la empresa, 1999.

sabana de Bogotá, no se encontraron diferencias estadísticamente significativas entre los períodos de rotación de los almacenistas $(p=0,39)$ y bomberos $(p=0,15)$, pero sí entre los asperjadores $(p=0,006)$.

Cuadro 2. Características del área de almacenamiento de plaguicidas.

\begin{tabular}{lcc}
\hline & \multicolumn{2}{c}{ Total } \\
\cline { 2 - 3 } Variable & $\begin{array}{c}\text { Número } \\
\text { N=83 }\end{array}$ & $\begin{array}{c}\text { Porcentaje } \\
\text { (\%) }\end{array}$ \\
\hline Área exclusiva & 69 & 82,1 \\
Señalizado & 23 & 27,4 \\
Cumple normas de almacenamiento & 47 & 55,9 \\
lluminación adecuada & 48 & 57,1 \\
Ventilación adecuada & 39 & 46,4 \\
Elementos para manejo de derrames & 67 & 79,7 \\
\hline
\end{tabular}

De las 84 empresas participantes con relación a la definición de los periodos de reentrada, algunas reportaron utilizar dos o más de los criterios seleccionados, $76(60,8 \%)$ lo hacen como política de la empresa, $42(32,6 \%)$ empresas tienen en cuenta la categoría toxicológica de los productos utilizados, $2(1,6 \%)$ siguen recomendaciones de las casas productoras y 3 $(2,4 \%)$ tienen en cuenta los parámetros establecidos por Florverde.

Al comparar los periodos de reentrada con los parámetros establecidos por Florverde según categoría toxicológica de los plaguicidas (categoría I: mínimo 24 horas; categoría ll: mínimo 12 horas; categoría III y IV: mínimo 6 horas) $(17,18)$, se encontró que $14(16,7 \%)$ empresas

Cuadro 3. Equipos empleados para la aplicación de plaguicidas por tamaño y ubicación geográfica de la empresa, Colombia, 2000.

\begin{tabular}{|c|c|c|c|c|c|c|c|}
\hline \multirow{2}{*}{$\begin{array}{l}\text { Tamaño } \\
\text { Ubicación del } \\
\text { equipo }\end{array}$} & \multicolumn{2}{|c|}{ Grande } & \multicolumn{2}{|c|}{ Mediana } & \multirow{2}{*}{$\begin{array}{c}\text { Pequeña } \\
\begin{array}{c}\text { Sabana } \\
(n=5)\end{array}\end{array}$} & \multicolumn{2}{|c|}{ Total } \\
\hline & $\begin{array}{c}\text { Antioquia } \\
\quad(n=6)\end{array}$ & $\begin{array}{c}\text { Sabana } \\
(n=34)\end{array}$ & $\begin{array}{c}\text { Antioquia } \\
\qquad(n=3)\end{array}$ & $\begin{array}{c}\text { Sabana } \\
(n=36)\end{array}$ & & $n=84$ & $\%$ \\
\hline Bomba de espalda & 5 & 27 & 3 & 27 & 5 & 67 & 79,7 \\
\hline Bomba estacionaria & 2 & 30 & 1 & 28 & 4 & 65 & 77,3 \\
\hline Bomba móvil & 6 & 34 & 3 & 34 & 1 & 78 & 92,8 \\
\hline Aguilón & 5 & 29 & 2 & 24 & 1 & 61 & 72,6 \\
\hline Aguilón de espalda & 0 & 2 & 0 & 1 & 0 & 3 & 3,6 \\
\hline Lanza & 5 & 33 & 2 & 33 & 5 & 78 & 92,9 \\
\hline
\end{tabular}


Cuadro 4. Uso de alternativas diferentes a los plaguicidas para el manejo de plagas y enfermedades empleadas por las empresas, Colombia, 2000.

\begin{tabular}{|c|c|c|c|c|c|}
\hline \multirow{2}{*}{$\begin{array}{l}\text { Tamaño } \\
\text { Ubicación } \\
\text { Tipo de control }\end{array}$} & \multicolumn{2}{|c|}{ Grande } & \multicolumn{2}{|c|}{ Mediana } & \multirow{2}{*}{$\begin{array}{c}\text { Pequeña } \\
\begin{array}{c}\text { Sabana } \\
(n=5)\end{array}\end{array}$} \\
\hline & $\begin{array}{c}\text { Antioquia } \\
(n=6)\end{array}$ & $\begin{array}{c}\text { Sabana } \\
(n=34)\end{array}$ & $\begin{array}{c}\text { Antioquia } \\
(n=3)\end{array}$ & $\begin{array}{c}\text { Sabana } \\
(n=36)\end{array}$ & \\
\hline Biológico & 6 & 25 & 3 & 18 & 3 \\
\hline Biorracional & 6 & 23 & 3 & 20 & 3 \\
\hline Métodos culturales & 6 & 31 & 3 & 23 & 3 \\
\hline Métodos físicos & 6 & 30 & 3 & 24 & 3 \\
\hline
\end{tabular}

Cuadro 5. Elementos de protección personal empleados por los asperjadores comparando la información de los trabajadores con el listado de verificación, Colombia 2000.

\begin{tabular}{|c|c|c|c|c|c|c|}
\hline \multirow[t]{2}{*}{ Elemento de protección personal } & \multicolumn{2}{|c|}{ Empresa } & \multicolumn{4}{|c|}{ Listado verificación } \\
\hline & No. & $\%$ & Sí & $\%$ & Mal uso & $\%$ \\
\hline Chaqueta impermeable & 83 & 98,8 & 81 & 96,4 & 2 & 2,4 \\
\hline Pantalón impermeable & 83 & 98,8 & 81 & 96,4 & 2 & 2,4 \\
\hline Peto & 8 & 1,2 & 1 & 1,2 & - & - \\
\hline Tyveck & - & - & 2 & 2,4 & - & - \\
\hline Pijama & 62 & 73,8 & 52 & 61,9 & 6 & 7,1 \\
\hline Respirador de doble cartucho & 57 & 67,9 & 36 & 42,9 & 11 & 13,1 \\
\hline Respirador de un cartucho & 24 & 28,6 & 27 & 32,1 & 7 & 8,3 \\
\hline Full-face & 4 & 4,8 & 4 & 4,8 & - & - \\
\hline Guantes cortos & 76 & 90,5 & 77 & 91,7 & 3 & 3,6 \\
\hline Guantos largos & 8 & 9,5 & 4 & 4,8 & - & - \\
\hline Mandriles - mangas & 1 & 1,2 & 2 & 2,4 & - & - \\
\hline Bota de caña alta & 82 & 97,6 & 79 & 94,0 & 1 & 1,2 \\
\hline Bota de caña baja & 2 & 2,4 & 4 & 4,8 & - & - \\
\hline Visor & 79 & 94,0 & 37 & 44,0 & 38 & 45,2 \\
\hline Monogafa & 3 & 3,6 & 4 & 4,8 & 1 & 1,2 \\
\hline
\end{tabular}

cumplen el tiempo establecido para la categoría I, $49(58,3 \%)$ para la categoría II, 46 (54,8\%) para la categoría III y $43(51,2 \%)$ empresas para la categoría IV.

Para el almacenamiento de los plaguicidas, del total de empresas participantes, $83(98,8 \%)$ reportaron contar con un lugar exclusivo para su almacenamiento. La empresa que no informó disponer de este lugar se encontraba en remodelación y estaba ubicada en la sabana de Bogotá. El cuadro 2 describe las características del área de almacenamiento de plaguicidas.

La distribución de los equipos utilizados para la aplicación de los plaguicidas por ubicación y tamaño de las empresas se presenta en el cuadro 3. Éstas pueden utilizar uno o más de los métodos descritos para este proceso.
Para el manejo de plagas y enfermedades, del total de empresas, $76(90,5 \%)$ utilizan alternativas diferentes a los plaguicidas. El cuadro 4 presenta el tipo de control empleado, por ubicación y tamaño de empresa.

Respecto a las alternativas empleadas teniendo en cuenta los diferentes tipos de control mencionados, las cinco más utilizadas fueron: erradicaciones en $82(97,6 \%)$ empresas, trampas en $51(60,7 \%)$, Trichoderma harzianum en 35 $(41,7 \%)$, extractos de ajo-ají en $32(38,1 \%)$ y extracto de tabaco en 26 (31\%). La mayoría de las empresas utilizan dos o más de los métodos descritos como alternativas para el control de plagas y enfermedades.

Con relación al uso de elementos de protección personal, en el cuadro 5 se muestra únicamente 
la de los asperjadores en forma comparativa teniendo en cuenta los elementos reportados por ellos mismos y los verificados por el equipo de trabajo de campo en cada una de las empresas participantes en el estudio.

Las pruebas de laboratorio más solicitadas a los trabajadores al ingresar a la empresa fueron: la de acetilcolinesterasa en $70(83,3 \%)$ empresas, serología para sífilis en $50(59,5 \%)$, y en $41(48,8 \%)$, la prueba de embarazo. Para el seguimiento de los trabajadores, 78 (92,9\%) empresas reportaron realizar pruebas de acetilcolinesterasa, de las cuales, 33 (42,3\%) llevan a cabo esta determinación antes de la exposición a cualquier tipo de plaguicidas (19 empresas grandes y 14 medianas), 9 (11,5\%) lo hacían después de la aplicación de productos organofosforados 0 carbamatos (4 grandes y 5 medianas), $17(21,8 \%)$ al finalizar la rotación por el área de aspersión (10 grandes y 7 medianas), y $44(56,4 \%)$ empresas las realizaban por calendario (19 grandes, 20 medianas y 5 pequeñas). Las empresas podían reportar una o más fechas para la toma de muestras, de conformidad a los programas de seguimiento médico establecidos por ellas.

Del total de pruebas de acetilcolinesterasa realizadas en 1999, $52(0,3 \%)$ se reportaron como anormales (1 en Antioquia y 51 en la sabana de Bogotá). Los métodos utilizados por los laboratorios para la determinación de los niveles de acetilcolinesterasa fueron: 67 (85,9\%) correspondieron al método de Michel-Aldrich modificado, $6(7,7 \%)$ a Merckotest y $5(6,4 \%)$ al método de Limperos y Ranta.

Algunas limitaciones del estudio incluyen la falta de aceptación de algunas empresas para participar en la investigación. Al inicio de la investigación se invitó a todas las empresas afiliadas a Asocolflores (204) a participar; 174 respondieron positivamente y fueron utilizadas como el universo del cual se hizo el muestreo representativo del total del sector. Adicionalmente, una vez se incluyeron las empresas que iban a ser objeto de la evaluación, fue necesario reemplazar la empresa no participante por otra empresa en forma aleatoria de la misma región y la misma categoría según el número de trabajadores. De igual manera, se procedió cuando el tamaño de la empresa en cuanto al número de trabajadores reportado en el listado inicial no coincidía con el informado en el momento de la visita de trabajo de campo. Otra limitante fue el no reporte del listado de consumo de plaguicidas por ingrediente activo por parte de 11 empresas (13,1\%). Sin embargo, al revisar la información de las empresas que no reportaron el listado de consumo de plaguicidas, no se encontraron diferencias al compararlas con las 73 empresas que reportaron esta información. Dentro de las razones que citaron dichas empresas fueron que contaban con la información de consumo de plaguicidas en formatos diferentes al proporcionado por Florverde y algunas empresas no tenían la información sistematizada.

\section{Discusión}

Del total de trabajadores que laboraban en las empresas participantes, se observó que la fuerza laboral en la Sabana en su mayoría es del sexo femenino, mientras que en Antioquia la mayor parte está representada por el sexo masculino. Los trabajadores de riesgo alto, los de riesgo medio y bajo pueden tener exposición a los plaguicidas en sus actividades normales de trabajo (jefes de área, operarios de campo, trabajadores en post-cosecha) ya que las desarrollan en un ambiente donde se manipulan estas sustancias (18). Se debe anotar que la clasificación de los trabajadores por grupo de riesgo utilizada en éste estudio, es arbitraria. Es posible que los trabajadores clasificados como de bajo riesgo, tengan exposición significativa a plaguicidas y en estudios futuros se debe determinar el nivel preciso de exposición que tienen, para poder establecer si dicha clasificación es precisa desde el punto de vista funcional.

Respecto a la categoría toxicológica, se observó que la mayoría de los plaguicidas utilizados son medianamente tóxicos, según la clasificación dada por el Ministerio de Salud de Colombia (15). Se encontraron diferencias estadísticamente significativas en el uso de plaguicidas entre Antioquia y la Sabana por categoría toxicológica y grupo químico, diferencias que podrían ser 
explicadas por el tipo de flor cultivada en cada región, ya que en Antioquia el principal cultivo es el pompón y en la Sabana, clavel y rosa, cultivos más sensibles al ataque de plagas y enfermedades.

Con base en la clasificación desde el punto de vista químico reportada por el Ministerio de Salud, Grupo de Protección de la Salud (comunicación escrita, Bogotá 2001), los plaguicidas más utilizados por las empresas para el control de plagas fueron los ditiocarbamatos seguidos por los metilisocianatos y los hidrocarburos clorados+organoclorados, entre otros. Los carbamatos y organofosforados correspondieron en conjunto al $7,9 \%$ ( $5,0 \%$ y $2,9 \%$ respectivamente) y los clorados al $0,11 \%$ del total de ingrediente activo/hectárea aplicado en las 1.238,6 hectáreas de las 73 empresas que reportaron el listado de consumo de plaguicidas del año 1999. La clasificación de los plaguicidas descrita en este estudio expresa únicamente el punto de vista químico pero no los aspectos de persistencia y acumulación, los cuales están íntimamente ligados al comportamiento de dichos compuestos en el medio ambiente (19).

Los períodos de rotación para los oficios de bombero y almacenista se encontraban entre 30 y 365 días. Los más prolongados se observaron en la Sabana. La estabilidad en cada oficio puede ser explicada por la experiencia adquirida en el manejo de plaguicidas en cada uno y por la capacitación impartida. Los asperjadores tenían períodos más cortos de rotación (7 a 180 días) debido a que la exposición a plaguicidas de éstos trabajadores se considera mayor. Es importante aclarar que no existen parámetros establecidos a nivel nacional con relación al tiempo de duración de los períodos de rotación.

Los periodos de re-entrada, o tiempo en el que se provee un margen de seguridad a los trabajadores después de una aspersión, varía de acuerdo a la categoría toxicológica del producto asperjado. Comparando los parámetros de los periodos de re-entrada descritos en el Programa Florverde con la información obtenida en el formulario de empresa, solamente $14(16,7 \%)$ de las empresas cumplían el tiempo establecido para la categoría
I, 49 (58,3\%) para la categoría II, 46 (54,8\%) para la categoría III y para la categoría IV $43(51,2 \%)$ empresas. Se debe resaltar que los tiempos descritos en el Programa Florverde difieren de los periodos de re-entrada establecidos por la Environmental Protection Agency (EPA) para los plaguicidas categoría I mínimo $48 \mathrm{~h}$, categoría II mínimo 24 h, categorías III y IV mínimo 12 h (20).

En cuanto al almacenamiento de los plaguicidas en las empresas participantes, en el $98,8 \%$ de estas, los plaguicidas son almacenados en áreas específicas para tal efecto. Sin embargo, se encontró una oportunidad para el mejoramiento significativo en el cumplimiento con lo dispuesto en la legislación vigente para el almacenamiento de plaguicidas, especialmente en la señalización, seguimiento general de las normas, iluminación, ventilación adecuadas y disponibilidad de elementos para el manejo de derrames (15).

Sólo el 3,6\% de las empresas participantes emplean el aguilón de espalda como equipo de aplicación de los plaguicidas. Considerando que desde el punto de vista de exposición del trabajador este equipo ofrece más garantías, ya que la nube de aspersión queda atrás del operario durante la aplicación, este bajo porcentaje podría ser explicado desde el punto de vista técnico y de infraestructura de los cultivos, ya que uno de los principales inconvenientes es el espacio de los caminos entre las camas, pues requiere un mínimo de $60 \mathrm{cms}$ para operarlo adecuadamente, por esto, los equipos más empleados para la aplicación de plaguicidas son aguilones convencionales y lanzas, produciendo mayor exposición al trabajador debido a que este va detrás de la nube de aspersión.

Respecto al uso de agroquímicos para el control de plagas y enfermedades de los cultivos, un alto porcentaje de empresas $(90,5 \%)$ ha incorporado alternativas diferentes al uso de plaguicidas para su control. Sin embargo, es necesario medir el impacto del uso de estos métodos respecto a la reducción del empleo de plaguicidas, el cual continúa siendo parte importante dentro de este manejo.

Con relación a los elementos de protección personal, la totalidad de las empresas reportaron 
suministrar los elementos necesarios para realizar los diferentes tipos de oficios. Sin embargo, no todos los trabajadores los tenían en buen estado al momento de la visita. Entre estos elementos los que se usan inadecuadamente son el visor, la monogafa, la pijama, el pantalón impermeable, el respirador de un cartucho y la chaqueta impermeable, lo que puede ocasionar un incremento potencial de la exposición a plaguicidas.

La prueba de laboratorio empleada al ingreso y para el seguimiento de los trabajadores con el objetivo de determinar la exposición a plaguicidas es la acetilcolinesterasa. Sin embargo, la mayoría de las empresas la usan de forma arbitraria para evaluar la exposición a todo tipo de plaguicidas y las muestras se toman del trabajador en puntos muy diversos dentro del proceso de producción de las flores. La utilización de la prueba de colinesterasa de esta forma no permite que se pueda justificar y evaluar su medición como biomarcador de exposición a plaguicidas organofosforados y carbamatos (21-25). Además, se usan otras pruebas diagnósticas no específicas para evaluar el efecto de los plaguicidas en la salud humana.

Se encontraron diferencias significativas en la práctica de la floricultura de acuerdo a la ubicación geográfica de las empresas. En la Sabana, los tipos de flor más cultivada son clavel y rosas, el proceso de clasificación de la flor se realiza en la poscosecha, el tamaño promedio de los invernaderos es de una hectárea y el estado fenológico y tipos de flor que se cultivan presentan gran uniformidad. Por el contrario, en Antioquia, el tipo de flor que más se siembra es el pompón, la clasificación se hace directamente en el cultivo, el tamaño de los invernaderos es mayor de una hectárea y los estados fenológicos son diferentes, lo que ocasiona que los trabajadores de esta zona tengan una exposición a plaguicidas diferente a los de la Sabana. No se encuentra una explicación clara que demuestre la mayor o menor eficiencia o las ventajas económicas que la práctica de la floricultura tenga, dependiendo de cual de los dos sistemas de producción se utilice, el de Antioquia o el de la Sabana de Bogotá. Indudablemente, tanto factores culturales, como la distribución de los trabajadores por sexo (masculino o femenino) en las dos regiones y otras variables que no se midieron en este estudio, podrían ayudar a explicar estas diferencias.

Los resultados de esta investigación se pueden generalizar solamente a las empresas afiliadas a Asocolflores. No podemos extrapolar los resultados a las empresas que no estén afiliadas, ya que no podemos determinar si tienen características similares.

A pesar de estas limitaciones, los resultados de nuestra investigación son representativos de las prácticas de la floricultura en las regiones estudiadas, las que incluyen la mayoría del área cultivada en el país.

Con base en los resultados obtenidos se sugieren las siguientes recomendaciones:

a) La utilización de productos de categoría I y II es significativa por lo que a corto plazo se debe lograr reducir al mínimo el uso de estos productos, haciendo un empleo racional de estos. Igualmente, se debe desarrollar un programa que resulte en la reducción en el uso de productos organoclorados o hidrocarburos clorados.

b) Debido a que no se están cumpliendo los tiempos de re-entrada establecidos por Florverde, es necesario el desarrollo de una investigación aplicada que permita establecer los períodos de rotación y tiempos de re-entrada adecuados para la actividad de la floricultura como se practica en Colombia. Las investigaciones para determinar los períodos de re-entrada y de rotación deben considerar la categoría toxicológica del producto, las condiciones atmosféricas de la región, el sexo del trabajador entre otras, ya que la absorción de los plaguicidas varía de acuerdo con estas variables.

c) Se deben adoptar las medidas recomendadas por el programa Florverde, respecto al almacenamiento y manejo de plaguicidas. Se recomienda a las empresas ajustar las condiciones de almacenamiento de los plaguicidas y de las unidades sanitarias, asegurándose de realizar lo establecido en el Decreto 1843, ya que el sector no está cumpliendo totalmente con la legislación vigente. 
d) Se deben adelantar investigaciones que resulten en el incremento de la utilización del aguilón de espalda y en la identificación de mecanismos que hagan dicho uso costo-efectivo para el floricultor, debido a que se requiere de un mínimo de espacio para la manipulación de dicho aguilón, equipo que de manera efectiva reduce la exposición del trabajador a los plaguicidas.

e) Es positivo el observar el uso de alternativas diferentes a agroquímicos, máxime si la introducción de estos métodos o alternativas resulta en una reducción en el uso de plaguicidas químicos, pero es necesario estrechar la vigilancia de los potenciales efectos adversos sobre la salud que pueden desencadenar los denominados productos biológicos, a través del desarrollo de investigaciones epidemiológicas.

f) Aún cuando se observa que las empresas proporcionan los elementos de protección personal necesarios para los diferentes oficios, un porcentaje de los trabajadores utiliza equipos que no se encuentran en buen estado y otra proporción los usa de manera incorrecta, por lo cual se recomienda de manera especial que las empresas diseñen estrategias de capacitación que generen cambios de comportamiento en el uso de estos elementos.

g) El valor de la actividad de la acetilcolinesterasa como biomarcador exclusivo de efecto a plaguicidas organofosforados y carbamatos, debe establecerse antes de la exposición a estas sustancias químicas y con al menos un seguimiento dentro de las 72 horas siguientes a la terminación de la exposición.

h) Las diferencias encontradas en los métodos de producción utilizados en las dos regiones del país estudiadas, sugieren la oportunidad de desarrollar un estudio de costo-efectividad comparando los dos modelos de producción. Además, dicha propuesta podría indicar cual sistema requiere un uso menor de plaguicidas de categoría toxicológica I y II.

Se debe estudiar la posibilidad de reducir el tamaño de los invernaderos e implementar métodos de control de la nube de aspersión dentro del área a tratar y buscar mayor uniformidad del estado fenológico en los cultivos de Antioquia, con el objeto de minimizar la exposición a plaguicidas de los trabajadores.

Las aspersiones y la aplicación de productos fitosanitarios deben hacerse de manera racional, con base en el conocimiento de plagas y enfermedades que se encuentran presentes en el cultivo. Una de las prácticas que se recomienda para tal efecto es la implementación y ejecución de un programa documentado de monitoreo de plagas y enfermedades, el cual debe elaborarse semanalmente.

i) Se sugiere a las empresas trabajar en los programas de capacitación y formación de los trabajadores en el manejo seguro de plaguicidas, asegurándose de dar cumplimiento a lo establecido en el decreto 1843.

j) Es urgente que se identifiquen recursos de todas las organizaciones privadas y públicas, nacionales e internacionales interesadas en la floricultura, la protección de los trabajadores y sus familias y del medio ambiente, para poder adelantar la investigación necesaria en Colombia que identifique los marcadores biológicos a utilizar para el seguimiento de los trabajadores expuestos a plaguicidas.

Nuestro estudio ha demostrado tener una utilidad inmediata para el sector y los trabajadores. $\mathrm{Ha}$ resultado en el desarrollo de sugerencias prácticas, algunas que se pueden implementar a corto y mediano plazo. Otras, requieren estrategias a más largo plazo y la identificación de recursos para ese propósito.

Este estudio permite hacer recomendaciones a los administradores, propietarios de las fincas y a los trabajadores, acerca de intervenciones que puedan reducir la exposición de la fuerza laboral, de una manera que sea costo-efectiva para la industria. Igualmente, se podrán planear y ejecutar campañas de educación para los administradores, los propietarios, los trabajadores y sus familias. Con base en los resultados de éste estudio, se podrán llevar a cabo estudios subsiguientes, en el tema de plaguicidas.

\section{Conflicto de intereses}

Como investigadores de este proyecto participaron un asesor de Asocolflores y un 
miembro del grupo de apoyo de Salud Ocupacional de esta misma empresa, quienes en conjunto con el resto de investigadores colaboraron desde la escritura del protocolo hasta la publicación de los resultados.

\section{Financiación}

Este proyecto fue financiado por el Instituto Nacional de Salud de Colombia, Universidad Thomas Jefferson, Filadelfia, PA, EEUU, Asociación Colombiana de Exportadores de Flores, Emory University, GA, EEUU y SURATEP.

\section{Agradecimientos}

Expresamos nuestros agradecimientos a los trabajadores, gerentes y propietarios de los cultivos participantes en el estudio, a los doctores Augusto Solano, Ángela María Orozco y a todos los funcionarios de Asocolflores por el apoyo técnico, logístico y administrativo. Alfonso Sarmiento de Suratep por su participación en el Comité Coordinador del programa de Investigación. En el Instituto Nacional de Salud a los doctores Moisés Wasserman, Jorge Boshell y Sandra Rocha por su valiosa colaboración durante el desarrollo del proyecto. A la Dra. Nelsy Rodríguez por el soporte en el análisis estadístico. Igualmente a Suratep y Asocolflores por el apoyo financiero y su asistencia con la organización de reuniones en Rionegro y Bogotá para la introducción del estudio y presentación de los resultados a los floricultores. Al doctor Samuel Henao, de la Organización Panamericana de la Salud y a la doctora María C. Leiva de la Universidad Thomas Jefferson, Filadelfia, PA, EEUU por sus comentarios al primer borrador de éste informe.

\section{Referencias}

1. Ministerio de Salud. Los plaguicidas en América Latina. Salud, ambiente y desarrollo. Segunda edición. Santafé de Bogotá: Ministerio de Salud; 1994.p.215-27.

2. O'Malley M. Clinical evaluation of pesticide exposure and poisonings. Lancet 1997;349:1161-6.

3. Blondell J. Epidemiology of pesticide poisonings in the United States, with special reference to occupational cases. Occup Med 1997;12:209-20.

4. Keifer MC, Mahurin RK. Chronic neurologic effects of pesticide overexposure. Occup Med 1997;12:291-304.
5. Zahm SH, Ward MH, Blair A. Pesticides and cancer. Occup Med 1997;12:269-89.

6. Sever LE, Arbuckle TE, Sweeney A. Reproductive and developmental effects of occupational pesticide exposure: the epidemiologic evidence. Occup Med 1997;12:305-25.

7. Restrepo M, Muñoz N, Day NE, Parra J, Hernandez C, Blettner M, et al. Birth defects among children born to a population occupationally exposed to pesticides in Colombia. Scand J Work Environ Health 1990;16:23946.

8. Restrepo M, Muñoz N, Day NE, Parra J, Romero L, Nguyen-Dinh $\mathbf{X}$. Prevalence of adverse reproductive outcomes in a population occupationally exposed to pesticides in Colombia. Scand J Work Environ Health 1990;16:232-8.

9. Varona M, De la Hoz F, Peña G, Cárdenas $\mathbf{O}$. Seguimiento de una cohorte de mujeres trabajadoras en cultivos de flores de la Sabana de Bogotá. Biomédica 1998;18:30-6.

10. Varona M, Cárdenas O, Crane C, Rocha S, Cuervo A, Vargas J. Prevalencia de alteraciones citogenéticas en trabajadores expuestos a plaguicidas en empresas de flores de Bogotá - Colombia. Biomédica 2003; 23:141-2.

11. Hoyos LS, Carvajal S, Solano L, Rodríguez J, Orozco L, López Y, et al. Cytogenetic monitoring of farmers exposed to pesticides in Colombia. Environ Health Perspect 1996;104 (Suppl 3):535-8.

12. Joksic G, Vidakovic A, Spasojevic-Tisma V. Cytogenetic monitoring of pesticide sprayers. Environ Res 1997;75:113-8.

13. Au WW, Wilkinson GS, Tyring SK, Legator MS, EI Zein R, Hallberg L, et al. Monitoring populations for DNA repair deficiency and for cancer susceptibility. Environ Health Persp 1996;104:579-84.

14. Lemeshow S, Hosmer DW, Klar J, Lwanga S. Adequacy of sample size in health studies. New York:John Wiley \& Sons;1990.p.45.

15. Ministerio de Salud. Disposiciones sanitarias sobre uso y manejo de plaguicidas. Decreto 1843 del 22 de julio de 1991. Bogotá: Ministerio de Salud;1991.p.1-69.

16. Asociación Colombiana de Exportadores de Flores (Asocolflores). Manejo adecuado de plaguicidas. Código de conducta. Autorregulación ambiental y social. Florverde. Manual de Mejores Prácticas. Versión 2.0 Bogotá;1998:060-13-060-15.

17. Oficina de Flower Label Programe (FLP). Pautas para una producción social y ambientalmente responsables de flores cortadas. Principios Básicos. Código de conducta internacional. Alemania; Publicado por Asocolflores:1999.

18. Torres C, Mora J, Ramírez G. Programa control de productos protectores de cultivo. Documento técnico. Santafé de Bogotá; Publicado por ARP Colpatria: 2000. 
19. Worthing $\mathbf{C}$, Hance $\mathbf{R}$. The pesticides manual. A world compendium. Ninth edition.Croydon:British Crop Protection Council:1991.p.1-1141.

20. United States Environmental Protection Agency EPA. Pesticides and toxic substances. Washington D.C.:EPA;1991.

21. Henao S, Corey G. Plaguicidas inhibidores de las colinesterasas. Serie Vigilancia 11. Centro Panamericano de Ecología Humana y Salud. Programa de Salud Ambiental. Organización Panamericana de la Salud. Organización Mundial de la Salud. Metepec, México: Editorial Clemente Aguilar;1991.p. 1-169.

22. Anwar WA. Biomarkers of human exposure to pesticides. Environ Health Perspect 1997;105 (Suppl 4):801-6.
23. De León J. Situación de las floricultoras con exposición crónica a mezclas de plaguicidas en Morelos, México. Boletín Rapam 1997;20:10-1.

24. Palacios ME, Paz P, Hernández S, Mendoza L. Sintomatología persistente en trabajadores industrialmente expuestos a plaguicidas organofosforados. Salud Publica Mex 1994;41:55-61.

25. Morgan DP. Diagnóstico y tratamiento de los envenenamientos por plaguicidas. Cuarta edición. Washington, D.C.;Health Effects Division, Office of Pesticide Programs, United States Environmental Protection Agency:1989.p. 1-11. 